\title{
Correction to: As-needed ICS-LABA in Mild Asthma: What Does the Evidence Say?
}

\author{
Christian Domingo ${ }^{1,2}\left(\mathbb{D} \cdot\right.$ Jordi Rello ${ }^{3} \cdot$ Ana Sogo $^{1,2}$
}

Published online: 29 October 2019

C) Springer Nature Switzerland AG 2019

\section{Correction to: Drugs (2019)}

https://doi.org/10.1007/s40265-019-01202-0

Abstract, fourth to last sentence, which currently reads:

Despite this poor evidence, and ignoring the clinical histological benefits of chronic inhaled corticosteroids (especially when administered promptly), GINA 2019 recently recommended daily low dose ICS or ICS-ICS as needed as a first option for step 2 patients.

Should read:

Despite this poor evidence, and ignoring the clinical histological benefits of chronic inhaled corticosteroids (especially when administered promptly), GINA 2019 recently recommended daily low dose ICS or ICS-LABA as needed as a first option for step 2 patients.

The original article has been corrected.

The original article can be found online at https://doi.org/10.1007/ s40265-019-01202-0.

Christian Domingo

cdomingo@tauli.cat

1 Servei de Pneumologia, Corporació Sanitària Parc Taulí, Parc Taulí s/n, 08208 Sabadell (Barcelona), Spain

2 Departament de Medicina, Universitat Autònoma de Barcelona (UAB), Barcelona, Spain

3 CIBERES, Instituto Salud Carlos III and Vall d'Hebron Institute of Research, Barcelona, Spain 